\title{
Spectroscopy of the quantum black hole
}

\author{
Jacob D. Bekenstein* \\ Racah Institute of Physics, Hebrew University of Jerusalem, Givat Ram, Jerusalem 91904, Israel \\ V. F. Mukhanov ${ }^{\dagger}$ \\ Institute of Theoretical Physics, ETH-Hoenggerberg, CH-8093 Zürich, Switzerland
}

(Received February 7, 2008)

\begin{abstract}
We develop the idea that, in quantum gravity where the horizon fluctuates, a black hole should have a discrete mass spectrum with concomitant line emission. Simple arguments fix the spacing of the lines, which should be broad but unblended. Assuming uniformity of the matrix elements for quantum transitions between near levels, we work out the probabilities for the emission of a specified series of quanta and the intensities of the spectral lines. The thermal character of the radiation is entirely due to the degeneracy of the levels, the same degeneracy that becomes manifest as black hole entropy. One prediction is that there should be no lines with wavelength of order the black hole size or larger. This makes it possible to test quantum gravity with black holes well above Planck scale.
\end{abstract}

*Electronic mail: bekenste@vms.huji.ac.il

†Electronic mail: mukhanov@itp.phys.ethz.ch 
In Hawking's conception [1] of the origin of black hole radiance, modes prepared in the pure vacuum state at $\mathcal{I}_{-}$contract, pass through the center of the collapsing star, and make it out to $\mathcal{I}_{+}$after spending a long time "skimming the horizon". The mode frequenies are redshifted down and the modes become populated by real quanta, the partners of those other members of the created pairs that have fallen through the horizon. The outgoing quanta have a thermal spectrum and statistics [2] that bear the imprint of passage through the potential barrier surrounding the black hole. The universality of this thermal character is viewed as due to the long sojourn of the emergent quanta close to the horizon.

Quantum fluctuations of the horizon, which are expected within any reasonable theory of quantum gravity, must change this simple picture. The Hawking quanta will not be able to "hover" at a nearly fixed distance from the horizon since the latter's very location has to fluctuate. Thus one suspects a modification of the character of the radiation when quantum gravity effects are properly taken into account, even for black holes very massive with respect to the Planck scale. In this letter we consider what modification of the naive Hawking radiance spectrum and statistics might reasonably be expected from quantum gravity.

Quantum systems of finite size more often than not display a discrete energy spectrum. Since the dynamics of a black hole responsible for its unique character refer to the finite region enclosed by the horizon, one may thus expect the mass spectrum of a black hole to display discreteness. Long ago the case was made [3, [4 that the black hole's horizon area should be quantized in integers. In particular (we use units with $G=c=1$ )

$$
A=\alpha \hbar n ; \quad n=1,2 \ldots
$$

where $\alpha$ is a pure number. This type of quantization law has since been considered by many workers [5.66. In particular, it is in agreement with the idea that area should be quantized in canonical quantum gravity [7]. The rule (1) implies that the mass of a nonrotating neutral black hole has a discrete spectrum. In general an energy level labeled by $n$ will be degenerate; let its multiplicity be denoted by $g(n)$. Further, it is natural to identify the entropy $S_{B H}=\frac{1}{4} A / \hbar+$ const. of the black hole in the level $n$ with $\ln g(n)$. The rule (1) in conjunction with the natural assumption that $g(1)=1$ or $S_{\mathrm{BH}}(n=1)=0$ (nondegenerate ground state) then forces us too choose $g(n)=e^{\alpha(n-1) / 4}$. But $g(n)$ must be integral, so $\alpha=4 \ln k$ where $k=2,3,4 \ldots$.

One of us [4] has argued that the choice $k=2$ is preferred because it would make the spacing in entropy of consecutive energy levels exactly one bit, an attractive value from the information-theoretic point of view. One convincing piece of evidence in favor of this choice is that it leads to $g(n)=2^{n-1}$ whereas the number of ways in which the black hole at level $n$ can be built starting from a situation with $A=0$ (no black hole) and going up the staircase of levels by various combinations of steps is also $2^{n-1}$. Thus for $k=2$ the degeneracy actually quantifyies the number of ways the black hole in the particular level could have formed. Put another way, the decay from the $n$-th level to the no-black hole state in such scheme can be accomplished in exactly $2^{n-1}$ sequences of steps [6]. There is thus weighty evidence in favor of $k=2$ or $\alpha=4 \ln 2$.

With this choice the energy spacing between consecutive levels for $M \gg \hbar$ corresponds to the (fundamental) frequency 


$$
\varpi=\frac{\ln 2}{8 \pi M}
$$

Clearly the radiation emitted as the black hole decays will be concentrated in lines at integer multiples of the fundamental frequency $\varpi$. Even if some broadening occurs or if the spectrum is blurred by two-quanta emission per jump, it is clear that the spectrum will be radically different from the one entertained in the standard discussion of Hawking radiance. One consequence of the physics here considered is that no radiation (or very little) should be emitted below $\varpi$. Observation of this type of spectrum for any black hole would immediately make quantum gravity effects observable well above the Planck scale. Herein lies the importance of our considerations.

Information about the intensity of the lines emitted by the black hole in the various particles available, and the statistics of these emissions can be obtained by first focusing on the de-excitation probabilities. We define these as follows. Describe the decay of the black hole during any interval of observer time $\Delta t$ by giving a sequence of integers $\left\{n_{1}, n_{2}, \cdots, n_{j}\right\}$. This means that during $\Delta t$ the black hole first jumped down $n_{1}$ elementary levels in one go, then $n_{2}$ levels, $\cdots$, and concluded by jumping down $n_{j}$ levels. In the process it emitted a quantum of some species of energy $n_{1} \hbar \varpi$, then a quantum of energy $n_{2} \hbar \varpi$, and so on.

These sequences can occur with any length $j$. The sequence of zero length, formally written $\{0\}$, represents the eventuality that the black hole did not decay at all in the interval $\Delta t$. One can associate with any sequence of length $j$ a conditional probability $P_{\Delta t}\left(\left\{n_{1}, n_{2}, \cdots, n_{j}\right\} \mid j\right)$ whose dependence on the species of quanta we ignore in this preliminary exploration. Obviously normalization requires that

$$
\sum_{\left\{n_{1}, n_{2}, \cdots, n_{j}\right\}} P_{\Delta t}\left(\left\{n_{1}, n_{2}, \cdots, n_{j}\right\} \mid j\right)=1
$$

where the all the $n_{k}$ are nonvanishing.

Even before dealing with the conditional probability $P_{\Delta t}\left(\left\{n_{1}, n_{2}, \cdots, n_{j}\right\} \mid j\right)$ given that the hole decayed by a sequence of $j$ jumps, we must evaluate the probability $p_{\Delta t}(j)$ for a sequence of exactly $j$ jumps (and $j$ emitted quanta) in time $\Delta t$. If $\Delta t$ is not vastly large, the black hole will have changed very little after time $\Delta t$ in terms of its emission properties (it will have nearly the same mass). Then the probabilities $p_{\Delta t}(j)$ for the next time interval $\Delta t$ can be taken as equal to the previous $p_{\Delta t}(j)$. On the basis of this and the fact that one does not attach significance to a failure of the hole to jump at some point in a sequence, we can write, for instance,

$$
p_{2 \Delta t}(1)=p_{\Delta t}(0) p_{\Delta t}(1)+p_{\Delta t}(1) p_{\Delta t}(0)=2 p_{\Delta t}(0) p_{\Delta t}(1)
$$

In words: a jump by one level in the interval $2 \Delta t$ can take place either by the jump taking place in the first half of the interval with no jump in the second half, or by no jump taking place in the first half with the jump by one step taking place in the second half. The above equality may be generalized to any odd $j$ by

$$
p_{2 \Delta t}(j)=2 p_{\Delta t}(0) p_{\Delta t}(j)+2 p_{\Delta t}(1) p_{\Delta t}(j-1)+\cdots+2 p_{\Delta t}(j / 2+1 / 2) p_{\Delta t}(j / 2-1 / 2)
$$

and to any even $j$ by 


$$
p_{2 \Delta t}(j)=2 p_{\Delta t}(0) p_{\Delta t}(j)+2 p_{\Delta t}(1) p_{\Delta t}(j-1)+\cdots+\left[p_{\Delta t}(j / 2)\right]^{2}
$$

These equations may be supplemented by the very obvious one

$$
p_{2 \Delta t}(0)=\left[p_{\Delta t}(0)\right]^{2}
$$

which just says that the survival probability of the black hole in a given level over time $2 \Delta t$ is product of the survival probability over time $\Delta t$ by itself. The solution of Eq. (7) is

$$
p_{\Delta t}(0)=e^{-\Delta t / \tau}
$$

where $\tau$ is a survival timescale to be determined. Substituting Eq. (8) into Eq. (4) gives a simple functional equation for $p_{\Delta t}(1)$ whose general solution is

$$
p_{\Delta t}(1)=\left(\Delta t / \tau^{*}\right) e^{-\Delta t / \tau}
$$

where $\tau^{*}$ is a positive constant-possibly distinct from $\tau$ - to be determined. Now substituting Eqs. (8)-(9) into Eq. (6) gives a functional equation for $p_{\Delta t}(2)$ which we solve by

$$
p_{\Delta t}(2)=(1 / 2)\left(\Delta t / \tau^{*}\right)^{2} e^{-\Delta t / \tau}
$$

On the basis of Eqs. (8)-(10) let us guess that for any $j$

$$
p_{\Delta t}(j)=(1 / j !)\left(\Delta t / \tau^{*}\right)^{j} e^{-\Delta t / \tau}
$$

To prove this guess for any odd $j$ we substitute Eq. (8) and our guess in Eq. (5), factor out the common factor $\left(\Delta t / \tau^{*}\right)^{j} e^{-j \Delta t / \tau}$, and multiply the resulting equation by $j$ ! to get

$$
2^{j-1}-1=j+\frac{j(j-1)}{2 !}+\frac{j(j-1)(j-2)}{3 !}+\cdots+\frac{j(j-1) \cdots(j / 2+1 / 2)}{2(j / 2) !}
$$

But this is plainly correct by the binomial expansion of $(1+1)^{j}$. Thus Eq. (11) is correct for all odd $j$. The proof of Eq. (11) for even $j$ proceeds similarly from Eq. (6). We must now check the normalization of $p_{\Delta t}(j)$ :

$$
\sum_{j} p_{\Delta t}(j)=\exp \left(\Delta t / \tau^{*}-\Delta t / \tau\right)
$$

Obviously we must set $\tau^{*}=\tau$ to get normalization.

Thus we have shown that the lengths of the sequence $\left\{n_{1}, n_{2}, \cdots, n_{j}\right\}$ (in other words, the number of quanta emitted during $\Delta t$ ) follows a Poisson probability distribution, [Eq. (11) with $\tau^{*}=\tau$ ] depending on a single parameter $\tau$. It is a consequence of Eq. (11) that the mean value of $j$ is simply $\langle j\rangle=\Delta t / \tau$.

Let us now return to the discussion preceding the introduction of $p_{\Delta t}(j)$. Given that the black hole has decayed during the interval $\Delta t$ by a sequence $\left\{n_{1}, n_{2}, \cdots, n_{j}\right\}$ of length $j$, what is the corresponding conditional probability distribution $P_{\Delta t}\left(\left\{n_{1}, n_{2}, \cdots, n_{j}\right\} \mid j\right)$ ? For $j=1$ the answer is not difficult to guess. When the black hole jumps $n_{1}$ steps down the ladder of levels, the degeneracy $g(n)$ changes by a factor of $2^{-n_{1}}$. If we assume the "matrix 
element" for a jump is the same for all $n_{1} \neq 0$, then the probability for the jump must be proportional to the final level's degeneracy. Thus

$$
P_{\Delta t}\left(\left\{n_{1}\right\} \mid 1\right) \propto 2^{-n_{1}}
$$

(The case $n_{1}=0$ is not included here because it belongs to $j=0$ ). From the normalization $\sum_{n_{1}=1}^{\infty} P_{\Delta t}\left(\left\{n_{1}\right\} \mid 1\right)=1$ we see immediately that the proportionality constant in Eq. (14) is unity. Combining Eq. (14) with Eq. (8) we get for the a priori probability that the hole jumps once $n_{1}$ steps in $\Delta t$

$$
P_{\Delta t}\left(\left\{n_{1}\right\}\right)=P_{\Delta t}\left(\left\{n_{1}\right\} \mid 1\right) p_{\Delta t}(1)=\left(\Delta t / 2^{n_{1}} \tau\right) e^{-\Delta t / \tau} .
$$

Now consider the case $j>1$. Up to now we have not regarded $\Delta t / \tau$ as having any particular value. However, it is clear from Eq. (11) that when $\Delta t \ll \tau, p_{\Delta t}(j)$ is very small for $j>1$. We are thus interested in calculating $P_{\Delta t}\left(\left\{n_{1}, n_{2}, \cdots, n_{j}\right\} \mid j\right)$ only for $\Delta t / \tau$ not small compared to unity. However, it is a useful strategy to imagine the interval $\Delta t$ divided into a large number $N \gg j$ of subintervals of equal durations $\epsilon \equiv \Delta t / N$. Because quantum transitions "do not take time", we can think of the $j$ jumps as taking place each in one of $j$ of the $N$ subintervals. There is no need to consider two or more jumps in one subinterval because, by our earlier remark, the probability of such an event would be very small because $N$ is large.

Now the $j$ "active" subintervals can be chosen out of the $N$ subintervals in a total of $N ! / j !(N-j) ! \approx N^{j} / j$ ! ways, where the approximation is a good one because $j \ll N$. Thus the analog of Eq. (15) is

$$
P_{\Delta t}\left(\left\{n_{1}, n_{2}, \cdots, n_{j}\right\}\right) \approx\left(N^{j} / j !\right) P_{\epsilon}\left(\left\{n_{1}\right\} \mid 1\right) P_{\epsilon}\left(\left\{n_{2}\right\} \mid 1\right) \cdots P_{\epsilon}\left(\left\{n_{j}\right\} \mid 1\right)\left[p_{\epsilon}(1)\right]^{j}\left[p_{\epsilon}(0)\right]^{N-j}
$$

where the last factor is the probability for the $N-j$ subintervals during which the hole does not decay. Substituting from Eq. (11) and replacing $\epsilon \Rightarrow \Delta t / N$ we have

$$
P_{\Delta t}\left(\left\{n_{1}, n_{2}, \cdots, n_{j}\right\}\right)=(1 / j !) 2^{-\left(n_{1}+n_{2}+\cdots+n_{j}\right)}(\Delta t / \tau)^{j} e^{-\Delta t / \tau}
$$

Under the stated assumptions this equation should be exact as $N \rightarrow \infty$. The set of probabilities is normalized in the sense that when summed for fixed $j$ over all configurations $\left\{n_{1}, n_{2}, \cdots, n_{j}\right\}$ with every $n_{i}$ starting from one, the result is exactly $p_{\Delta t}(j)$, so that the further sum over $j$ yields unity. Another thing to notice is that

$$
P_{\Delta t}\left(\left\{n_{1}, n_{2}, \cdots, n_{j}\right\} \mid j\right) \equiv P_{\Delta t}\left(\left\{n_{1}, n_{2}, \cdots, n_{j}\right\}\right) / p_{\Delta t}(j)=2^{-\left(n_{1}+n_{2}+\cdots+n_{j}\right)} .
$$

Thus given that the black hole jumped $j$ times in $\Delta t$, the distribution of the sizes of the jumps is independent of $\Delta t$ as well as any scale set by the black hole. (In fact, we could obtain Eq. (18) directly by repeating our argument that if the transition matrix element is constant, the probabilities depend only on the multiplicity of the final level.) One consequence of this is that the spectrum and the statistics of the radiation are steady so long as the mass of the hole changes by little.

Let us now estimate the parameter $\tau$. The conditional probabilities Eq. (18) are normalized. Since $\left\langle n_{i}\right\rangle=\sum_{n_{i}=1}^{\infty} n_{i} 2^{-n_{i}}=2$, each one of the $j$ quanta carries energy $2 \hbar \varpi$ on 
average. Since the mean value of $j$ is $\Delta t / \tau$, we see that the mean decrease in black hole mass during $\Delta t$ is just $2 \hbar \varpi \Delta t / \tau$ so that

$$
d\langle M\rangle / d t=-2 \hbar \varpi \Delta t / \tau
$$

Due to the thermal character of the radiation, we may estimate $d\langle M\rangle / d t$ by using the Stefan-Boltzmann law for a surface of area $16 \pi\langle M\rangle^{2}$ at temperature $(\hbar / 8 \pi)\langle M\rangle^{-1}$ :

$$
\frac{d\langle M\rangle}{d t}=-\frac{\gamma \hbar}{1530 \pi\langle M\rangle^{2}}
$$

Here $\gamma$ accounts for two fact: the radiation comes out only in lines and not over the entire black-body spectrum, and the emission is by a variety of particles. The first fact tends to force $\gamma$ under unity, while the second promotes a value well above unity. On the balance we feel $\gamma$ should be regarded as being unity to within an order of magnitude. Inserting the value of $\varpi$ and comparing these equations gives us

$$
\tau=3840 \gamma^{-1}\langle M\rangle \ln 2
$$

It is plain that $\tau$, the mean time between quantum leaps, is large compared to the dynamical black hole timescale $\langle M\rangle$.

We now calculate the probability distribution $p_{\Delta t}\left(k \mid n_{k} \varpi\right)$ that in the course of time $\Delta t$, the black hole emits $k$ quanta of frequency $n_{k} \varpi$. If the hole makes $j$ jumps in $\Delta t$, the number of ways of selecting the $k$ quanta out of the $j$ is $C_{k}^{j} \equiv j ![k !(j-k) !]^{-1}$, and the probability of each such selection is $2^{-\left(n_{1}+n_{2}+\cdots+n_{j-k}\right)}\left(2^{-n_{k}}\right)^{k}$ with none of the $n_{1}, n_{2}, \cdots, n_{j-k}$ agreeing with $n_{k}$. We must sum the product over all allowed values of $n_{1} \neq n_{k}, n_{2} \neq n_{k}$, etc. Since $\sum_{n=1}^{\infty} 2^{-n}=1$, the sum over each of the $j-k$ factors $2^{-n_{i}}$ gives a factor $1-2^{-n_{k}}$. Thus

$$
p_{\Delta t}\left(k \mid n_{k} \varpi, j\right)=\frac{j !}{k !(j-k) !}\left(1-\frac{1}{2^{n_{k}}}\right)^{j}\left(\frac{1}{2^{n_{k}}-1}\right)^{k}
$$

We now multiply this conditional probability by the absolute probability distribution $p_{\Delta t}(j)$ in Eq. (11), and sum over all $j \geq k$. Since $\sum_{j=s}^{\infty} x^{j} /(j-s) !=e^{x} x^{s}$, we have again a Poisson distribution:

$$
p_{\Delta t}\left(k \mid n_{k} \varpi\right)=(1 / k !)\left(x_{n_{k}}\right)^{k} e^{-x_{n_{k}}} ; \quad x_{n} \equiv(\Delta t / \tau) 2^{-n} .
$$

Is this result not at variance with ones conception of black hole radiation as thermal ? For thermal radiation the number of quanta in a given mode is distributed exponentially; here we have a Poisson distribution. However, the discrepancy is merely a result of the different sort of question we ask here: how is the number of quanta emitted during a time interval in all modes of frequency $n_{k} \varpi$ distributed ?

To see that the Poisson distribution which answers this question is consistent with a thermal distribution, consider a blackbody cavity at temperature $T$. Let us look at all quanta contained in a random subvolume. Each quantum drawn from that subvolume is subject to a Boltzmann distribution; accordingly, the probability that we draw one quantum of frequency $\omega$ is $A \exp (-\hbar \omega / T)$ where $A$ is a normalization constant. Succesive drawings are independent drawings from the same distribution. Thus the probability that we draw exactly $k$ quanta 
of frequency $\omega_{k}$ from a series of $j$ drawings is $C_{k}^{j} A^{k} \exp \left(-k \hbar \omega_{k} / T\right) A^{j-k} \Pi_{i=1}^{j-k} \exp \left(-\hbar \omega_{i} / T\right)$ summed over all possible values of the frequencies $\omega_{i}$ distinct from $\omega_{k}$. By normalization $A \sum_{\omega} \exp (-\hbar \omega / T)=1$, so if the $\omega_{k}$ is missing from the sum, the corresponding factor in the product, after the summation, will be $1-A \exp \left(-\hbar \omega_{k} / T\right)$. We get for $k$ a probability distribution exactly like Eq. (22) with the replacement $2^{-n_{k}} \rightarrow A \exp \left(-\omega_{k} / T\right)$. The perfect analogy dispels the impression that the distribution for the black hole radiance is not thermal.

Another interesting question is what is the probability that $k_{1}$ quanta are emitted into one group of modes, all of frequency $n_{k} \varpi$, and $k_{2}$ into an equal number of modes of the same frequency (distinguished from the first group, say, by angular momentum) ? Since there are $C_{k_{1}+k_{2}}^{k_{1}}$ ways to divide $k_{1}+k_{2}$ quanta of frequency $n_{k} \varpi$ into one class with $k_{1}$ and one with $k_{2}$, and a total of $2^{k_{1}+k_{2}}$ such bipartitions,

$$
p_{\Delta t}\left(k_{1}, k_{2} \mid n_{k} \varpi\right)=p_{\Delta t}\left(k_{1}+k_{2} \mid n_{k} \varpi\right) 2^{-\left(k_{1}+k_{2}\right)} C_{k_{1}}^{k_{1}+k_{2}} .
$$

With help of Eq. (23) this takes the form

$$
p_{\Delta t}\left(k_{1}, k_{2} \mid n_{k} \varpi\right)=p_{\Delta t / 2}\left(k_{1} \mid n_{k} \varpi\right) p_{\Delta t / 2}\left(k_{2} \mid n_{k} \varpi\right)
$$

That is, the probabilities for $k_{1}$ and $k_{2}$ are independent and of Poisson type, but their means are, naturally, reduced to half of that for $k_{1}+k_{2}$. The generalization to the probability for many sets of modes of equal frequencies is obvious.

Let us now inquire into the probability $p_{\Delta t}\left(k, l \mid n_{k} \varpi, n_{l} \varpi\right)$ for the emission of $k$ quanta of frequency $n_{k}$ and $l$ quanta of different frequency $n_{l}$ in time $\Delta t$. There are $j ![k ! l !(j-l-k) !]^{-1}$ ways for the $k$ and $l$ quanta to be chosen - without regard to identity-from the $j$. The probability of each such choice is $2^{-\left(n_{1}+n_{2}+\cdots+n_{j-k}\right)}\left(2^{-n_{k}}\right)^{k}\left(2^{-n_{l}}\right)^{l}$. We sum the product over all allowed values of $n_{1} \neq n_{k}$ and $n_{1} \neq n_{l}$, etc. By the logic already invoked, the sum over $n_{i}$ of each of the $j-k-l$ factors $2^{-n_{i}}$ is replaced by a factor $1-2^{-n_{k}}-2^{-n_{l}}$. Instead of Eq. (22) we get

$$
p_{\Delta t}\left(k, l \mid n_{k} \varpi, n_{l} \varpi, j\right)=\frac{j !}{k ! l !(j-k-l) !}\left(1-\frac{1}{2^{n_{k}}}-\frac{1}{2^{n_{k}}}\right)^{j}\left(\frac{1}{2^{n_{k}}}-1\right)^{k}\left(\frac{1}{2^{n_{l}}}-1\right)^{l}
$$

We now multiply by $p_{\Delta t}(j)$ of Eq. (11), and sum over all $j \geq k+l$ to get the result

$$
p_{\Delta t}\left(k, l \mid n_{k} \varpi, n_{l} \varpi\right)=p_{\Delta t}\left(k \mid n_{k} \varpi\right) p_{\Delta t}\left(l \mid n_{l} \varpi\right)
$$

Thus the probabilities for emission into the two frequencies are independent and of Poisson type. The generalization of this result to $n$ frequencies is obvious.

We conclude by commenting on the shape of the spectrum. The mean value of the number of quanta emitted at a given frequency $\omega=n \varpi$ is, according to Eq. (23), proportional to $\Delta t$ and to $2^{-n}$. Since emissions at diverse frequencies are independent, it follows that the intensity of the line at frequency $n \varpi$ must be proportional to $n 2^{-n}$ or equivalently to $\omega e^{-8 \pi M \omega}$. In accordance with our earlier assumption, this is independent of particle species. The spectrum is to be compared with the Planckian spectrum (phase space factor aside) $\omega /\left(e^{\hbar \omega / T}-1\right)$. The black hole spectrum is obviously similar to that of thermal radiation of like temperature in a finite box. 
We found earlier that the rate of emission of quanta is $\tau^{-1}$. Thus the width of the lines in frequency should be of order $\tau^{-1}$. But we concluded that $\tau$ is between a few hundreds and a few tens of thousands of $M$. We thus expect the width of the lines in frequency to be small compared to $M^{-1}$, which is well below the size of the frequencies, Eq. (2). Therefore, the various black hole lines are unlikely to overlap; the black hole spectrum must be clearly discrete. This conclusion differs from Hawking's original prediction even for massive black holes. Fluctuations of the horizon are here seen as manifested in a discretization of the radiance's spectrum. No quanta are expected with frequencies below $M^{-1}$. These predictions will become amenable to experimental check if primordial black holes are ever found. They open a window upon quantum gravity that does not require reaching down to Planck scale physics.

V. M. is grateful to the Racah Institute, Hebrew University, for hospitality. J. D. B.'s work is partly supported by a grant from the Israel Science Foundation, administered by the Israel Academy of Sciences and Humanities. 


\section{REFERENCES}

[1] S. W. Hawking, Commun. Math. Phys. 43, 199 (1975).

[2] L. Parker, Phys. Rev.D12, 1519 (1975); J. D. Bekenstein, Phys. Rev.D12, 3077 (1975); R. M. Wald, Commun. Math. Phys. 43, 199 (1975).

[3] J. D. Bekenstein, Lett. Nuovo Cimento 11, 467 (1974).

[4] V. Mukhanov, Pis. Eksp. Teor. Fiz. 44, 50 (1986) [JETP Letters 44, 63 (1986)], and in Complexity, Entropy and the Physics of Information, SFI Studies in the Sciences of Complexity, vol. III, ed. W. H. Zurek (Addison-Wesley, New York 1990).

[5] Yu. I. Kogan, Pis. Eksp. Teor. Fiz. 44, 209 (1986) [JETP Letters 44, 267 (1986)]; M. Schiffer, "Black Hole Spectroscopy" (unpublished); J. Bellido, "Quantum Black Holes", report hep-th/9302127 (unpublished); M. Maggiore, Nucl. Phys. B429, 205 (1994); Y. Peleg, report hep-th 9307057 (unpublished); C. Lousto, (unpublished).

[6] U. H. Danielsson and M. Schiffer, Phys. Rev. D48, 4779 (1993).

[7] A. Ashtekar, C. Rovelli and L. Smolin, Phys Rev Letters 69, 237 (1992). 